\title{
Variation of crystal orientation in a single crystal copper by nano plastic forming
}

\author{
Masahiko YOSHINO ${ }^{1}$, Nayuta MINAMI ${ }^{2}$, Hidehiko KIMURA ${ }^{3}$ \\ Takashi MATSUMURA ${ }^{4}$, Noritsugu UMEHARA ${ }^{5}$ \\ ${ }^{1}$ Department of Mechanical and Control Engineering, Tokyo Institute of Technology, Japan, \\ myoshino@mes.titech.ac.jp \\ ${ }^{2}$ Department of Mechanical and Control Engineering, Tokyo Institute of Technology, \\ ${ }^{3}$ EcoTopia Science Institute, Nagoya University, Japan \\ ${ }^{4}$ Department of Mechanical Engineering, Tokyo Denki University, Japan \\ ${ }^{5}$ Department of Mechanical Science and Engineering, Nagoya University, Japan
}

\begin{abstract}
:
This paper reports studies on new nano fabrication technology to control crystalline structure of metallic material. Wedge tool indentation tests were conducted on a single crystal copper specimen by using nano forming tester, and crystal rotation by plastic deformation was studied. Cross sections of indent grooves made by nano forming tester were machined by FIB, and distribution of crystal orientation on the cross section was analyzed by the method of EBSD. Results revealed that crystal rotation around an indent groove is separated into two zones, and rotation axis correspond with [100] direction regardless of crystal orientation and tool direction. This finding show possibility of crystalline structure control of metallic materials by nano plastic forming technique.
\end{abstract}

Keywords: Nano/micro fabrication, Single crystal copper, Crystal rotation, Deformation characteristics

\section{Introduction}

Recent attempt to develop new nano/micro devices such as MEMS and micro TAS requires new fabrication technology available for various materials. Photo lithography method has been often utilized for manufacturing nano/micro structures on silicon wafer or glass plates. Photo lithography method, however, is not always suitable for research and development of newer devices due to restriction on work material, cost, stringency in method etc. In order to overcome these disadvantages, newer fabrication technologies, such as nano imprinting method, have been studied.

The authors have proposed a nano fabrication process by direct imprinting method, which is named the nano plastic forming. This is a so simple process that a specially designed tool is imprinted on the surface on the work material to duplicates its nano/micro structure. Since this is a plastic forming process in principle, it has many advantages such as high thorough put, low emission, flexibility in geometry, and variation of work material. Plastic forming process also has another advantage that it can control microstructure of metallic material. It is known that plastic deformation stores energy in crystals of a work material, and the stored energy induces metallurgical phenomena like recrystallization when heat treatment is applied. Such metallurgical phenomena results in improvement of material properties. Nano plastic forming is effective to apply deformation energy to a crystal of a work material because size of deformation by nano plastic forming (about $1-10 \mu \mathrm{m}$ ) is smaller than average grain size of metal crystals. Therefore combination of nano plastic forming and heat treatment has possibility in developing new nano/micro devices where crystalline structure is controlled.

In order to realize this technology, it is necessary to understand mechanism of crystal deformation around tool indent. Since a single crystal has anisotropic mechanical property, plastic deformation by tool indentation cannot be explained by conventional isotropic theory, i.e. Mises theory. Many researchers have been studying meso-plasticity theory based on the assumption of slip model of crystalline structure. However, only a few experimental studies have been done on crystal deformation, especially on the nano plastic forming process.

This paper reports a new experimental technique to study detail of crystal deformation under nano plastic forming. Wedge type tool was utilized for the indentation test, which is a typical model studied on plasticity theory. The paper also presents some typical results on crystal rotation induced by the tool indentation.

\section{Experimental method}

\subsection{Experimental device}

Figures 1 illustrate a nano plastic forming tester used for the experiments. It has computer controlled $X-Y$ stages and a $\mathrm{Z}$ stage. Resolution of those stages is $10 \mathrm{~nm}$, 
and the stroke of $\mathrm{X}-\mathrm{Y}$ stages is $20 \mathrm{~mm}$ whereas for $\mathrm{Z}$ stage, the stroke is of $40 \mathrm{~mm}$. A tool and a load cell are mounted on the $\mathrm{Z}$ stage. The load cell measures the indentation load. The complete process of imprinting involving the movement of stages and imprinting load are controlled by a computer. The nano plastic forming test is conducted inside a clean booth so that effect of dust is avoided


Figure 1 Nano plastic forming tester

Figure 2 shows a knife edge tool used for the experiment. This is made of a single crystal diamond. Edge angle is 60 degrees, and the width is $0.6 \mathrm{~mm}$. Its edge is ground to very sharp and the edge radius is less than $50 \mathrm{~nm}$.



Figure 2 Knife edge tool made of single

\subsection{Single crystal copper specimens}

Single crystal copper was used as work material. Rectangle specimens as shown in figure 3 were cut out from a single crystal ingot. The upper surface of the specimen was polished to mirror like, and finished by chemical polishing so that distorted layer is removed. Three kinds of specimens of various crystal orientations were prepared; where $\{100\},\{110\}$ or $\{111\}$ crystal plane corresponded to the upper surface respectively, and [100] or [110] crystal axes corresponded with the edge of the specimen.

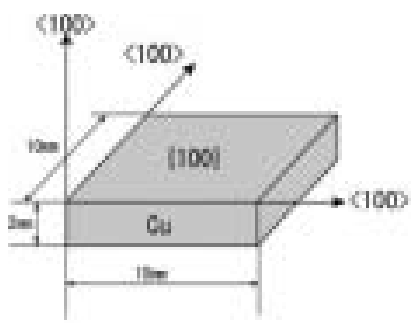

Figure 3 Geometry of a single crystal specimen.



(a) $\{001\}$



(b) $\{110\}$

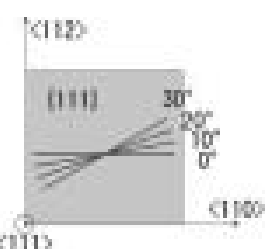

(c) $\{111\}$
Figure 4 Tested tool directions

\subsection{Experimental procedure}

The knife edge tool was indented to the specimens at the indentation load of $2.0 \mathrm{~N}$. The indentation tests were conducted on several tool directions as shown in the figures 4 . These tool directions were determined based on symmetry of the crystal orientation.

After the indentation, EBSD specimens were cut out from the indent cross section. The specimen is machined by FIB as shown in the figure 5 (a), where the cross section of the indent groove corresponds with the surface of the EBSD specimen. Then the EBSD specimen was picked up, and fixed on a specimen holder as shown by figure 5 (b). The coordinate system on the EBSD specimen is shown in the figure.

The EBSD specimen was applied to the EBSD analysis, and crystal orientation around the indent groove was measured.
( a)



(b)



Figure 5 EBSD specimen cut from the indented specimen.

\section{Experimental results}

\subsection{EBSD analysis}

Figures 6 shows examples of distribution of crystal orientation around a indent groove. The specimen is of $\{100\}$. The figures indicate crystal plans orienting to ND direction by color shown in the standard triangle of the inverse pole figure (e). It is found from these figures that crystal orientation was changed by tool indentation, and distribution of variation of crystal orientation depends on the tool direction.



(a)Tool direction: $0^{\circ}$



(c)Tool direction: $30^{\circ}$



(b) Tool direction: $15^{\circ}$



(d)Tool direction: $45^{\circ}$



(e)Standard triangle of the inverse pole figure.

Figure 6 Crystal orientation around indent grooves. 


\subsection{Distribution of spin vectors}

In order to clarify crystal rotation obtained by the EBSD analysis, spin vector of crystal at each measured point is calculated by the following method.

First, determine the initial crystal orientation tensor $\boldsymbol{R}_{\boldsymbol{0}}$, which corresponds with the original crystal orientation of the specimen. Then, calculate a crystal orientation tensor after deformation $\boldsymbol{R}_{\boldsymbol{1}}$ from the Euler angle obtained by EBSD analysis. The spin tensor of crystal rotation $\boldsymbol{W}$ is related to $\boldsymbol{R}_{\boldsymbol{0}}$ and $\boldsymbol{R}_{\boldsymbol{I}}$ with the following equation.

$$
\boldsymbol{R}=\left\{\boldsymbol{I}+\boldsymbol{W} d t+\frac{1}{2 !}(\boldsymbol{W} d t)^{2}+\frac{1}{3 !}(\boldsymbol{W} d t)^{3} \cdots \frac{1}{n !}(\boldsymbol{W} d t)^{n}\right\} \boldsymbol{R}_{0}
$$

where the spin tensor has components shown as follows,

$$
\boldsymbol{W}=\left[\begin{array}{ccc}
0 & -\omega_{3} & \omega_{2} \\
\omega_{3} & 0 & -\omega_{1} \\
-\omega_{2} & \omega_{1} & 0
\end{array}\right]
$$

The vector composed with $\left(\omega_{1}, \omega_{2}, \omega_{3}\right)$ results in the spin vector, which indicates rotation axis, and whose magnitude expresses rotation angle.

The equation is solved by the simplex method where $\delta$ in the following equation is optimized.

$$
\begin{aligned}
& \delta=\sum_{i, j=1}^{3} \Delta_{i j}{ }^{2} \\
& \Delta\left(\omega_{1}, \omega_{2}, \omega_{3}\right)= \\
& \boldsymbol{R}-\left\{\boldsymbol{I}+\boldsymbol{W} d t+\frac{1}{2 !}(\boldsymbol{W} d t)^{2}+\frac{1}{3 !}(\boldsymbol{W} d t)^{3} \cdots \frac{1}{n !}(\boldsymbol{W} d t)^{n}\right\}
\end{aligned}
$$

Figures 7 illustrate distribution of the rotation angle (magnitude of the spin vectors) analyzed by the proposed method. These are examples of indentation tests on the $\{001\}$ plane. These figures show crystal rotation occurred around the indent grooves. It is found that rotated region is small when the tool direction is 0 degree, whereas the rotated region extends deep in the specimen when the tool direction is 45 degree.

Distribution of crystal rotation is separated into two zones; upper zone and lower zone for both side of the indent groove. The largest crystal rotation appears in the flank of the indent groove in the upper zone.



(a) Tool direction: $0^{\circ}$



(c) Tool direction: $30^{\circ}$



(b) Tool direction: $15^{\circ}$

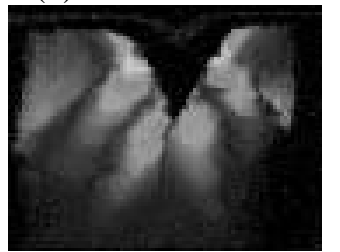

(d) Tool direction: $45^{\circ}$



$21^{\circ}$
Figure 7 Distribution of the crystal rotation angle around indent grooves on $\{100\}$ plane.

Figure 8 shows distribution of the vector direction angle $\theta$, which is defined as the angle between the spin vector and [100] axis. The vector direction angle $\theta$ is calculated by the following equation.

$$
\theta=\cos ^{-1}\left(\frac{\boldsymbol{\omega} d t \bullet \boldsymbol{n}_{r e f}}{|\boldsymbol{\omega} d t|\left|\boldsymbol{n}_{r e f}\right|}\right)
$$

where $\boldsymbol{\omega} d t$ is a spin vector at each measured point and $\boldsymbol{n}_{r e f}$ is reference vector ([100] direction in this case). These figures show that most of the analyzed region is occupied with region of $\theta=0$ degree or region of $\theta=180$ degrees, and region of intermediate angle is small. This implies that most of spin vectors indicate [100] direction or [-100] direction.

By comparing figures 7 and figures 8 , it is found that the upper zone rotates to inside direction, whereas the lower zone rotates to the outside direction. Figure 9 illustrates this model schematically, where Zone I indicates the upper zone, whereas Zone II indicates the lower zone.

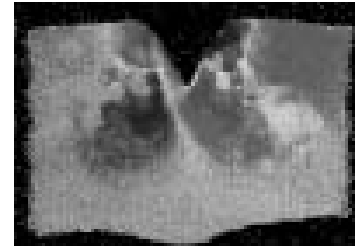

(a) Tool direction: $0^{\circ}$



(c) Tool direction: $30^{\circ}$ $180^{\circ}$ $135^{\circ}$ $90^{\circ}$



(b) Tool direction: $15^{\circ}$



(d) Tool direction: $45^{\circ}$ $45^{\circ}$ 0

Figure 8 Distribution of the vector direction angles $\theta$ around indent grooves on $\{100\}$ plane.



Figure 9 Schematic illustration of crystal rotation by a wedge type tool indentation.

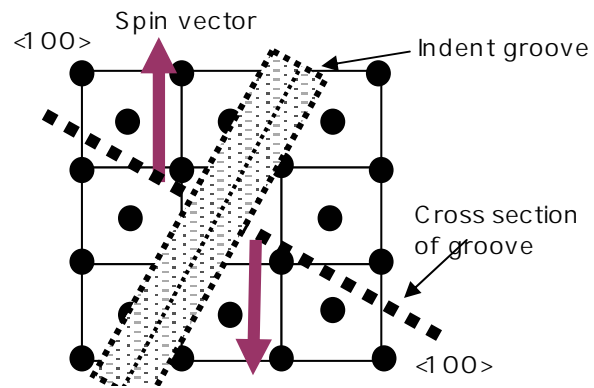

Figure 10 Schematic illustration of spin vectors around an indent, which indicates $<100\rangle$ direction. 
Important finding is that this pattern of crystal rotation does not depend on the tool direction. All of figures in figure 7 and figure 8 show similar characteristics on rotation distribution. Most of all spin vectors indicates [100] direction or [-100] direction regardless of tool direction. Figure 10 illustrate geometrical relationship between the tool direction (groove direction) and spin vector. When these spin vectors are observed on the cross section, symmetric distribution as seen in figure 8 appears.

\subsection{Effect of crystal orientation of specimen}

Figures 11-14 compare distributions of the crystal rotation angle and $\theta$ around grooves indented on $\{110\}$ plane and $\{111\}$ plane. All of these figures show that distribution of crystal rotation is separated into two zones as the same as seen in figure 7 and figure 8 . Distributions of vector direction angle $\theta$ are also divided into two regions; 0 degree and 180 degrees. This result indicates Zone I and Zone II are also formed even though crystal orientation is different. These results also imply that most of spin vector indicate $\langle 100\rangle$ direction or $<-100\rangle$ direction regardless of crystal orientation.



(a) Tool direction: $0^{\circ}$



(c) Tool direction: $60^{\circ}$



(b) Tool direction: $35.5^{\circ}$

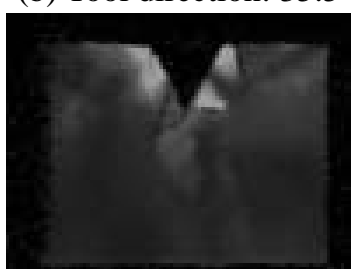

(d) Tool direction: $90^{\circ}$
Figure 11 Distribution of the crystal rotation angle around indent grooves on $\{110\}$ plane.


(c) Tool direction: $60^{\circ}$



(b) Tool direction: $35.5^{\circ}$

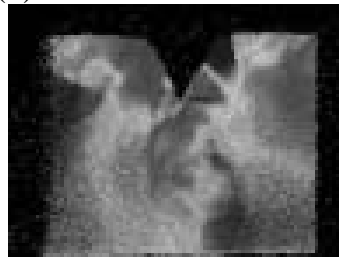

(d) Tool direction: $90^{\circ}$
Figure 12 Distribution of the $\theta$ around indent grooves on $\{110\}$ plane.

\section{Conclusion}

Crystal rotation induced by nano plastic forming is studied in this paper. Concluding remarks are summarized as follows,
(1) Nano plastic forming tests were conducted on single crystal copper specimens. Crystal orientation around a indent groove is analyzed by a new experimental technique using FIB machining and EBSD analysis.

(2) Spin vector of crystal rotation is calculated by tensor analysis using the EBSD data. The spin vector indicates axis of crystal rotation, and its magnitude expresses rotation angle.

(3) It is found that rotated region is separated into two zones, upper zone (Zone I) and lower zone (Zone II). Zone I rotates to the inside direction, and Zone II rotates to the outside direction.

(4) Most of spin vectors indicates [100] or [-100] directions regardless of the tool direction and crystal orientation of specimen.

\section{Acknowledgement}

This research is supported by the Grant-in-Aid for Scientific Research (16360063) of JSPS. It is also supported by AMADA foundation and SUZUKI foundation. The authors would like appreciate to their contribution.



(a) Tool direction: $0^{\circ}$



(c) Tool direction: $20^{\circ}$



(b) Tool direction: $10^{\circ}$



(d) Tool direction: $30^{\circ}$ Figure 13 Distribution of the crystal rotation angle around indent grooves on $\{111\}$ plane.



(a) Tool direction: $0^{\circ}$



(c) Tool direction: $20^{\circ}$



(b) Tool direction: $10^{\circ}$



(d) Tool direction: $30^{\circ}$

Figure 14 Distribution of the $\theta$ around indent grooves on $\{111\}$ plane. 